\title{
Global existence of weak solutions for the Navier-Stokes equations with capillarity on the half-line
}

\author{
Zhong Tan and Rongcong Guo
}

\begin{abstract}
In this paper, we construct the global weak solutions to the initial-boundary problem for the Navier-Stokes system with capillarity in the half space $\mathbb{R}_{+}^{1}$. The result extends Eugene Tsyganov's existence theorem which considered the problem in the finite region published in J. Differential Equaions 245:3936-3955, 2008.
\end{abstract}

Mathematics Subject Classification (2000). 35A01, 35A24, 35D30, 74G25.

Keywords. Navier-Stokes equations, Half space problem, Global weak solution, Capillarity.

\section{Introduction}

We are concerned with compressible fluids endowed with internal capillarity. The model we consider originates from the XIXth century work by Van der Waals and Korteweg [13] and was actually derived in its modern form in the 1980s using the second gradient theory, see for instance $[11,14,15]$.

Korteweg-type models are based on an extended version of nonequilibrium thermodynamics, which assumes that the energy of the fluid not only depends on standard variables but also on the gradient of the density. Let us now consider a fluid of density $\rho \geq 0$, velocity field $u \in \mathbb{R}^{n}$, we are now interested in the following compressible capillary fluid model, which can be derived from a Cahn-Hilliard like free energy (see the pioneering work by Dunn and Serrin [7] and also in $[1,5,8])$. The conservation of mass and of momentum write: 


$$
\left\{\begin{array}{l}
\partial_{t} \rho+\operatorname{div}(\rho u)=0 \\
\partial_{t}(\rho u)+\operatorname{div}(\rho u \otimes u+p I)=\operatorname{div}(K+S)
\end{array}\right.
$$

where the viscous stress tensor $S$ and the Korteweg stress tensor $K$ are given by

$$
\left\{\begin{array}{l}
S=\lambda \operatorname{div}(u I)+\mu\left(\partial_{i} u_{j}+\partial_{j} u_{i}\right) \\
K=\left(\kappa \rho \Delta \rho+\frac{1}{2}\left(\kappa^{\prime} \rho+\kappa\right)|\nabla \rho|^{2}\right) I-\kappa \nabla \rho \otimes \nabla \rho
\end{array}\right.
$$

and where $\kappa$ is the coefficient of capillarity and is a regular function. The term $\operatorname{div} K$ allows to describe the variation of density at the inter faces between two phases, generally a mixture liquid-vapor. $p$ is a general pressure term. $\mu$ and $\lambda$ are the Lamé viscosity coefficients depending on the density $\rho$ and satisfying $\mu>0$ and $2 \mu+n \lambda \geq 0$.

We now mention some results concerning the Korteweg type system. Danchin, R., Desjardins study the well-posedness of the model in spaces with critical regularity indices with respect to the scaling of the associated equations. And in a functional setting as close as possible to the physical energy spaces, they prove global existence of solutions close to a stable equilibrium, and local in time existence for solutions when the pressure law may present spinodal regions. Uniqueness is also obtained in [6].

Bresch et al. [2] consider a global existence result of weak solutions in dimension 2 or 3 using the regularity of the density in a periodic domain $\Omega=T^{d}$. Next they discuss the case of a smooth bounded domain depending on the choice of the diffusive term and on the boundary conditions, with a positive result in the case $\Omega=(0,1) \times T^{d-1}$. Third they also indicate some partial results on the associated shallow water equation that means the system corresponding to $\kappa=0$.

Haspot [4] improve the existence of global weak solution in dimension two for initial data in the energy space, close to a stable equilibrium and with specific choices on the capillary coefficients. In particular they are interested in capillary coefficients approximating a constant capillarity coefficient $\kappa$. Third they show the existence of global weak solution in dimension one for a specific type of capillary coefficients $\kappa=\frac{1}{\rho^{2+\varepsilon}} 1_{\{\rho \leq \alpha\}}+\theta(\rho) 1_{\{\alpha<\rho \leq 2 \alpha\}}+\kappa 1_{\{\rho>2 \alpha\}}$ with large initial data in the energy space.

In the one-dimensional space with the other specific type of capillary coefficients $\kappa=\rho^{-5}$, Tsyganov [16] proved the global existence and asymptotic convergence of weak solutions on the Lagrangian coordinate by a easier method different with Kato [12] and Bona and Sachs [3]. And Tsyganov study the model in finite interval $[0,1]$. And we extend the existence result to the half space situation.

In this paper we prove the existence of global weak solutions for the Navier-Stokes equations with capillarity

$$
\begin{aligned}
v_{t}-u_{x} & =0, \quad x \in[0, \infty), t>0, \\
u_{t}+p(v)_{x} & =\left(\frac{u_{x}}{v}\right)_{x}-v_{x x x}, \quad x \in[0, \infty), t>0,
\end{aligned}
$$


with Cauchy data

$$
(v(x, 0), u(x, 0))=\left(v_{0}(x), u_{0}(x)\right)
$$

such that

$$
\left(v_{0}-v^{\prime}\right) \in H^{1}([0, \infty)), \quad u_{0} \in L^{2}([0, \infty))
$$

and the boundary conditions

$$
v_{x}(0, t)=u(0, t)=0 .
$$

where $v, u$, and $p$ are the specific volume, velocity, and pressure in the fluid, $v^{\prime}>0$ is a constant, and (1.1) and (1.2) are respectively the equations of conservation of mass and balance of forces. We assume throughout that

$$
p(v)=v^{-\gamma}, \quad \gamma>1
$$

For convenience, we define a special energy function $\psi(v)$ by

$$
\psi(v):=\int_{v^{\prime}}^{v} p\left(v^{\prime}\right)-p(s) d s
$$

where $v^{\prime}>0$ is fixed. We then have some properties with $\psi(v)$ in following

$$
\psi(v)=p\left(v^{\prime}\right)\left(v-v^{\prime}\right)+\frac{v^{1-\gamma}-\left(v^{\prime}\right)^{1-\gamma}}{\gamma-1} \geq 0
$$

as a obvious conclusion of (1.6), we have

$$
\psi(v) \sim v^{1-\gamma}, \quad \text { as } v \rightarrow 0 ; \text { and } \psi \sim v, \quad \text { as } v \rightarrow \infty
$$

and when $\psi$ blow-up

$$
\lim _{v \rightarrow+0} \psi(v)=+\infty, \quad \lim _{v \rightarrow+\infty} \psi(v)=+\infty
$$

easy to see that $\psi\left(v^{\prime}\right)=\psi^{\prime}\left(v^{\prime}\right)=0$ and $\psi^{\prime \prime}(v)=-\psi^{\prime}(v)>0$. Thus there exist $C>0$ depending only on the pressure $p$,

$$
C^{-1}\left(v-v^{\prime}\right)^{2} \leq \psi(v) \leq C\left(v-v^{\prime}\right)^{2}, \quad 0<\underline{\mathrm{v}} \leq v \leq \bar{v} .
$$

We say that $(v, u)$ is a weak solution of (1.1)-(1.3), provided that $\inf _{x \in[0, \infty)} v(\cdot, t)>0$ and

$$
\begin{aligned}
& v(\cdot, t) \in C\left([0, \infty) ; H^{1}([0, \infty))\right) \cap C\left((0, \infty) ; H^{2}([0, \infty))\right), \\
& u(\cdot, t) \in C\left([0, \infty) ; L^{1}([0, \infty))\right) \cap C\left((0, \infty) ; H^{1}([0, \infty))\right), \\
& v(\cdot, 0)=v_{0}, \quad u(\cdot, 0)=u_{0}, \quad v_{x}(0, t)=u(0, t)=0 .
\end{aligned}
$$

and for all test functions $\phi \in C_{0}^{1}((0, \infty) \times[0, \infty)), \varphi \in C_{0}^{3}((0, \infty) \times[0, \infty))$, and all times $t_{2}>t_{1} \geq 0$. 


$$
\begin{gathered}
\left.\int_{0}^{\infty} v \phi d x\right|_{t_{1}} ^{t_{2}}-\int_{t_{1}}^{t_{2}} \int_{0}^{\infty}\left(v \phi_{t}-u \phi_{x}\right) d x d t=0 \\
\left.\int_{0}^{\infty} u \varphi d x\right|_{t_{1}} ^{t_{2}}-\int_{t_{1}}^{t_{2}} \int_{0}^{\infty}\left(u \varphi_{t}+p(v) \varphi_{x}\right) d x d t \\
=-\int_{t_{1}}^{t_{2}} \int_{0}^{\infty}\left(\frac{u_{x}}{v} \varphi_{x}-v \varphi_{x x x}\right) d x d t
\end{gathered}
$$

Our existence result is formulated in the following theorem:

Theorem 1. Assume that the initial data $\left(v_{0}, u_{0}\right)$ satisfy

$$
\begin{gathered}
0<\underline{v} \leq v_{0}(x) \leq \bar{v} \\
\int_{0}^{\infty}\left(\frac{1}{2} u_{0}^{2}(x)+\psi\left(v_{0}(x)\right)+\frac{1}{2} v_{0}^{2}(x)\right) d x=C_{0},
\end{gathered}
$$

for some positive constants $\underline{v}, \bar{v}, C_{0}$. Then there is a weak solution $(v, u)$ of (1.1)-(1.3) and positive constant $C(T)$ depending only on $\underline{v}, \bar{v}, C_{0}$ and $T$, such that

$$
\begin{gathered}
0<C(T)^{-1} \leq v(x, t), \quad 0 \leq t \leq T \\
\sup _{0 \leq t \leq T}\left(\int_{0}^{\infty}\left(v(x, t)-v^{\prime}\right)^{2} d x+\int_{0}^{\infty} v_{x}(x, t)^{2} d x+\int_{0}^{\infty} u(x, t)^{2} d x\right. \\
\left.+t \int_{0}^{\infty} u_{x}(x, t)^{2} d x\right) \leq C(T) .
\end{gathered}
$$

\section{Difference approximations}

In this section we construct approximate solutions to the system (1.1)-(1.5) and show that they satisfy various regularity estimates. We employ the method of semi-discrete difference schemes which can be considered standard for Navier-Stokes equations in Lagrangean coordinates. In fact, our analysis shares many ideas with the one in Hoff $[9,10]$.

Let $h$ be an increment in $x$, which be small enough. We set $x_{k}=k h$ for $k \in \mathbb{Z}_{+} \cup\{0\}$, and $x_{j}=j h, j+1 / 2 \in \mathbb{Z}_{+} \cup\{0\}$. Define the difference operator $\delta$ by

$$
\delta w_{l}:=\frac{w_{l+1 / 2}-w_{l-1 / 2}}{h}
$$

for $l \in \mathbb{Z} \cup \mathbb{Z}+\frac{1}{2}$. We then use the following semi-discrete scheme to generate approximations $v_{j}(t)$ and $u_{k}(t)$ :

$$
\begin{gathered}
\dot{v}_{j}=\delta u_{j}, \quad j=\frac{1}{2}, \frac{3}{2}, \frac{5}{2}, \cdots \\
\dot{u}_{k}+\delta p(v)_{k}=\delta\left(\frac{\delta u}{v}\right)_{k}-\delta(\delta(\delta v))_{k}, \quad k=0,1,2,3, \ldots \\
\left(v_{j}(0)-v^{\prime}\right), \quad\left(u_{k}(0)\right) \in l^{2} \cap l^{\infty},
\end{gathered}
$$


where $v^{\prime}>0$ be a positive constant, denote $\dot{w}:=\partial_{t} w$. It is implied that $v_{j}$ and $u_{k}$ in the system (2.1)-(2.3) depend also on $h$, but we will drop the superscript $h$ throughout the section.

Lemma 2.1. Assume that the equations (2.1)-(2.3) have a solution defined up to time $T$ with $v_{j}(t)>0$ for $0 \leq t \leq T$, Then for $0 \leq t \leq T$ :

$$
\begin{aligned}
I(t):= & \frac{1}{2} \sum_{k=0}^{+\infty} u_{k}^{2}(t) h+\sum_{j=\frac{1}{2}}^{+\infty} \psi\left(v_{j}(t)\right) h+\frac{1}{2} \sum_{k=0}^{+\infty} \delta v_{k}^{2}(t) h \\
& +\int_{0}^{t} \sum_{j=\frac{1}{2}}^{+\infty} \frac{\delta u_{j}^{2}}{v_{j}} h=I(0)=C
\end{aligned}
$$

Proof. Differentiating $I(t)$ with respect to $t$, rearranging, and applying equations (2.1) to $(2.3)$

$$
\begin{aligned}
\dot{I}(t)= & \sum u_{k} \dot{u}_{k} h+\sum \delta v_{k} \delta \dot{v}_{k} h+\sum\left(p\left(v^{\prime}\right)-p\left(v_{j}\right)\right) \dot{v}_{j} h+\sum \frac{\delta u_{j}^{2}}{v_{j}} h \\
= & \sum u_{k} \dot{u}_{k} h-\sum \delta(\delta v)_{j} \delta u_{j} h+\sum p\left(v^{\prime}\right) \delta u_{j} h+\sum u_{k} \delta p_{k} h \\
& -\sum u_{k}\left(\frac{\delta u}{v}\right)_{k} h \\
= & \sum u_{k} \dot{u}_{k} h+\sum_{k} \delta(\delta(\delta v))_{k} u_{k} h+\sum p\left(v^{\prime}\right) \delta u_{j} h+\sum u_{k} \delta p_{k} h \\
& -\sum u_{k}\left(\frac{\delta u}{v}\right)_{k} h \\
= & \sum u_{k}\left(\dot{u}_{k}+\delta p(v)_{k}-\delta\left(\frac{\delta u}{v}\right)_{k}+\delta(\delta(\delta v))_{k}\right) h+p\left(v^{\prime}\right) \sum \delta u_{j} h \\
= & 0
\end{aligned}
$$

This proved (2.4).

From Lemma 2.1, for fixed $h$, and the property (1.8) of $\psi$, we obtain

$$
\left|u_{k}\right| \leq C(h), 0<C^{-1}(h) \leq v_{j} \leq C(h), \text { uniformly for all } k, j \text { and } t \geq 0
$$

and so the ode system (2.1) to (2.3) can be solved globally. Next we'll prove that there is a boundary of $v_{j}$, which is independent on $h$.

Lemma 2.2. There is a solution $\left(v_{j}(t), u_{k}(t)\right)$ of $(2.1)-(2.3)$, defined for all $t>0$, satisfying

$$
\sum_{j}\left(v_{j}(t)-v^{\prime}\right)^{2} \leq C, \quad \text { and } \quad 0<\frac{1}{C(T)} \leq v_{j}(t), 0 \leq t \leq T
$$

Proof. The first term of (2.5) is a direct conclusion of (2.4) applying (1.9), and we'll prove the last term of (2.5) by three claims below: 
Claim 2.2.1. For fix $j\left((j+1 / 2) \in \mathbb{Z}_{+}\right)$, then for each $t \geq 0$, there is an integer $j^{\prime}=j^{\prime}(j, t)$ depending measurably on $t$, such that:

$$
\begin{aligned}
& \sum_{l=j+1}^{j^{\prime}(j, t)} v_{l}(t) h \geq 1 \\
& v_{j^{\prime}(j, t)} \geq \frac{1}{C}>0 \\
& \left|x_{j^{\prime}}-x_{j}\right| \leq C .
\end{aligned}
$$

Proof of claim 2.2.1. Fix $t_{0}>0$ choose first $j^{\prime \prime}>j$ such that:

$$
\sum_{l=j+1}^{j^{\prime \prime}-1} v_{l}\left(t_{0}\right) h \leq 1<\sum_{l=j+1}^{j^{\prime \prime}} v_{l}\left(t_{0}\right) h
$$

then choose $j^{\prime} \geq j^{\prime \prime}$ be most close to $j^{\prime \prime}$, so that: $v_{j^{\prime}}\left(t_{0}\right) \geq \min \left(\frac{1}{2}, \frac{v^{\prime}}{2}\right)$. We will get an upper bounded for $\sum_{l=j+1}^{j^{\prime}} v_{l}\left(t_{0}\right) h$. First:

$$
\sum_{l=j^{\prime \prime}}^{j^{\prime}-1} v_{l}\left(t_{0}\right) h \leq \sum_{l=j^{\prime \prime}}^{j^{\prime}-1} \frac{1}{2} h=\frac{1}{2}\left|x_{j^{\prime}-1}-x_{j^{\prime \prime}}\right| \leq \frac{1}{2}\left|x_{j^{\prime}}-x_{j}\right|
$$

next: let $l=j^{\prime}$ or $j^{\prime \prime}$, if $v_{l} \leq v^{\prime}+1$ then $v_{l} h \leq C$, otherwise if $v_{l}>v^{\prime}+1$ then by the definition of $\psi(v)$ and Lemma 2.1

$$
v_{l} h \leq C \psi\left(v_{l}\right) h \leq C
$$

therefore:

$$
1<\sum_{l=j+1}^{j^{\prime}(j, t)} v_{l}(t) h \leq C+\frac{1}{2}\left|x_{j^{\prime}}-x_{j}\right|
$$

(2.9) together with $v_{j^{\prime}}\left(t_{0}\right) \geq \min \left(\frac{1}{2}, \frac{v^{\prime}}{2}\right) \geq \frac{1}{C}>0$ enable us to define $j^{\prime}$ as a measurable function of $t$.

We observe that: $\frac{\gamma}{\gamma-1}$ and $\gamma$ are conjugate indices. Using holder inequality we have that:

$\left|x_{j^{\prime}}-x_{j}\right|=\sum_{l=j+1}^{j^{\prime}} 1 \cdot h=\sum_{l=j+1}^{j^{\prime}} v_{l}^{\frac{\gamma-1}{\gamma}} v_{l}^{\frac{1-\gamma}{\gamma}} h \leq\left(\sum_{l=j+1}^{j^{\prime}} v_{l} h\right)^{\frac{\gamma-1}{\gamma}}\left(\sum_{l=j+1}^{j^{\prime}} v_{l}^{1-\gamma} h\right)^{\frac{1}{\gamma}}$ by the definition of $\psi$, we know $\psi(v) \sim v^{1-\gamma}$ as $v \rightarrow 0$, so $v^{1-\gamma} \leq C \psi(v)+$ $1, v>0$ and then we get:

$$
\begin{aligned}
\left|x_{j^{\prime}}-x_{j}\right| & \leq\left(C+\frac{1}{2}\left|x_{j^{\prime}}-x_{j}\right|\right)^{\frac{\gamma-1}{\gamma}}\left(\sum_{l=j+1}^{j^{\prime}}\left(C \psi\left(v_{l}\right)+1\right) h\right)^{\frac{1}{\gamma}} \\
& \leq\left(C+\frac{1}{2}\left|x_{j^{\prime}}-x_{j}\right|\right)^{\frac{\gamma-1}{\gamma}}\left(C+\left|x_{j^{\prime}}-x_{j}\right|\right)^{\frac{1}{\gamma}}
\end{aligned}
$$

this proves (2.8). 
Claim 2.2.2. For fix $j$ and $t \in[0, T]$ and let $j^{\prime}=j^{\prime}(j, t)$ as in claim 2.2.1, then for $l<j^{\prime}$ :

$$
v_{l}(t) A(t)=\frac{v_{l}(0)+I_{l}(t)}{v_{j^{\prime}(j, t)}(0) B_{l}(t)}
$$

where

$$
\begin{array}{r}
A(t)=\frac{\left.\exp \left(\int_{0}^{t} p_{j^{\prime}(j, t)}(\tau)+\delta(\delta v)_{j^{\prime}(j, t)}(\tau) d \tau\right)\right)}{v_{j^{\prime}(j, t)}(t)} \\
B_{l}(t)=\exp \left(\sum_{l<k<j^{\prime}(j, t)}\left(u_{k}(t)-u_{k}(0)\right) h\right) \\
I_{l}(t)=\int_{0}^{t} v_{j^{\prime}(j, s)}(0) A(s) B_{l}(s) v_{l}(s)\left(p_{l}(s)+\delta(\delta v)_{l}(s)\right) d s .
\end{array}
$$

Proof of claim 2.2.2. Denote $E(v)=\ln v, E_{j}=E\left(v_{j}\right)$ and then by the equation (2.2) and (2.1),

$$
\begin{aligned}
\delta \dot{E}_{k}= & \delta\left(\frac{\delta u}{v}\right)_{k}=\dot{u}_{k}+\delta p_{k}+\delta(\delta(\delta v))_{k} \\
\left.\delta E_{k}(\cdot)\right|_{0} ^{s}= & \left.u_{k}(\cdot)\right|_{0} ^{s}+\int_{0}^{s}\left(\delta p_{k}+\delta(\delta(\delta v))_{k}\right)(\tau) d \tau \\
\Rightarrow & \left.\left(E_{j^{\prime}(j, s)}(\cdot)-E_{l}(\cdot)\right)\right|_{0} ^{s}=\left.\sum_{l<k<j^{\prime}(j, s)} \delta E_{k}(\cdot)\right|_{0} ^{s} h \\
= & \left.\sum_{l<j^{\prime}(j, s)} u_{k}(\cdot)\right|_{0} ^{s} h+\int_{0}^{s}\left(p_{j^{\prime}(j, s)}(\tau)-p_{l}(\tau)\right) d \tau \\
& +\int_{0}^{s}\left[\delta(\delta v)_{j^{\prime}(j, s)}(\tau)-\delta(\delta v)_{l}(\tau)\right] d \tau \\
= & \ln B_{l}(s)+\int_{0}^{s}\left(p_{j^{\prime}}(j, s)(\tau)-p_{l}(\tau)\right) d \tau \\
& +\int_{0}^{s}\left[\delta(\delta v)_{j^{\prime}(j, s)}(\tau)-\delta(\delta v)_{l}(\tau)\right] d \tau \\
\Rightarrow & -E_{l}(s)+\int_{0}^{s}\left[p_{l}(\tau)+\delta(\delta v)_{l}(\tau)\right] d \tau \\
= & -E_{j^{\prime}(j, s)}(s)+E_{j^{\prime}(j, s)}(0)-E_{l}(0)+B_{l}(s) \\
& +\int_{0}^{s}\left[p_{j^{\prime}(j, s)}(\tau)+\delta(\delta v)_{j^{\prime}(j, s)}(\tau)\right] d \tau
\end{aligned}
$$

take exponentials, we then get:

$$
\begin{aligned}
\frac{\left.\exp \left(\int_{0}^{s} p_{l}(\tau)+\delta(\delta v)_{l}(\tau) d \tau\right)\right)}{v_{l}(s)} & =\frac{\left.v_{j^{\prime}(j, s)}(0) \exp \left(\int_{0}^{s} p_{j^{\prime}(j, s)}(\tau)+\delta(\delta v)_{j^{\prime}(j, s)}(\tau) d \tau\right)\right)}{v_{l}(0) v_{j^{\prime}(j, s)}(s)} B_{l}(s) \\
& =\frac{v_{j^{\prime}(j, s)}(0)}{v_{l}(0)} A(s) B_{l}(s)
\end{aligned}
$$


multiplying by $v_{l}(s)\left(p_{l}(s)+\delta(\delta v)_{l}(s)\right)$, we then get:

$\left.\frac{d}{d s} \exp \left(\int_{0}^{s} p_{l}(\tau)+\delta(\delta v)_{l}(\tau) d \tau\right)\right)=\frac{v_{j^{\prime}(j, s)}(0)}{v_{l}(0)} A(s) B_{l}(s) v_{l}(s)\left(p_{l}(s)+\delta(\delta v)_{l}(s)\right)$

Thus:

$$
\left.\exp \left(\int_{0}^{t} p_{l}(s)+\delta(\delta v)_{l}(s) d s\right)\right)=1+\frac{I_{l}(t)}{v_{l}(0)}=\frac{v_{l}(0)+I_{l}(t)}{v_{l}(0)}
$$

Substituting this into (2.11) and rearranging, we then obtain (2.10).

Pointwise bounds for $v_{j}(t)$ will follow esily from (2.10) once we have estimated the quantities $A(t)$ and $B_{l}(t)$ appeared in claim 2.2.2.

Claim 2.2.3. For $j \leq l \leq j^{\prime}(j, t)$, there holds:

$$
\begin{aligned}
0 & <\frac{1}{C} \leq B_{l}(t) \leq C \\
A(t) & \leq C(t) .
\end{aligned}
$$

Proof of claim 2.2.3.

$$
\begin{aligned}
& \left|\sum_{l<k<j^{\prime}(j, t)}\left(u_{k}(t)-u_{k}(0)\right) h\right| \leq C\left(\sum_{l<k<j^{\prime}(j, t)}\left(u_{k}(t)^{2}+u_{k}(0)^{2}\right) h\right)^{1 / 2}\left(\sum_{l<k<j^{\prime}(j, t)} 1^{2} h\right)^{1 / 2} \\
& \quad \leq C \cdot\left|x_{j^{\prime}}-x_{j}\right|^{1 / 2} \leq C
\end{aligned}
$$

this implies (2.12).

Next to prove (2.13), by (2.6)

$$
\begin{aligned}
A(t) & \leq A(t) \sum_{l=j+1}^{j^{\prime}(j, t)} v_{l}(t) h=\sum_{l=j+1}^{j^{\prime}(j, t)}\left(A(t) v_{l}(t)\right) h=\sum_{l=j+1}^{j^{\prime}(j, t)} \frac{v_{l}(0)+I_{l}(t)}{v_{j^{\prime}(j, t)}(0) B_{l}(t)} h \\
& \leq C \sum_{l=j+1}^{j^{\prime}(j, t)}\left(v_{l}(0)+I_{l}(t)\right) h
\end{aligned}
$$

by the definition of $\psi$, we know $v \leq C(\psi(v)+1)$ when $v>0$, so

$$
\sum_{l=j+1}^{j^{\prime}(j, t)} v_{l}(0) h \leq C\left(1+\left|x_{j^{\prime}}-x_{j}\right|\right) \leq C
$$

and by the initial condition $v_{j}(0) \leq \bar{v}$ and (2.12), we get

$$
I_{l}(t) \leq C \int_{0}^{t} A(s) v_{l}(s)\left(p_{l}(s)+\delta(\delta v)_{l}(s)\right) d s
$$

so that (2.14) becomes:

$$
A(t) \leq C+C \int_{0}^{t} A(s)\left(\sum_{l=j+1}^{j^{\prime}(j, t)} v_{l}(s)\left(p_{l}(s)+\delta(\delta v)_{l}(s)\right) h\right) d s
$$


and if we know that

$$
\sum_{l=j+1}^{j^{\prime}(j, t)} v_{l}(s)\left(p_{l}(s)+\delta(\delta v)_{l}(s)\right) h \leq C
$$

then call for the Gronwall's inequality to (2.15) would proves (2.13). And we observe

$$
\left|\sum_{1 / 2}^{\infty} \delta(\delta v(s))_{l} v_{l}(s) h\right|=\left|-\sum_{0}^{\infty} \delta v_{k}(s)^{2} h\right| \leq C
$$

by the properity of convergent serise we get $\left|\sum_{j+1}^{j^{\prime}(j, t)} \delta(\delta v(s))_{l} v_{l}(s) h\right| \leq C(T)$, so we leave to prove

$$
\sum_{1 / 2}^{\infty} p_{j}(s) v_{j}(s) h \leq C
$$

For this aim, let's multiplying $(2.2)$ by $u_{k}$ and rearrange

$$
\frac{d}{d t} \frac{u_{k}^{2}}{2}+u_{k} \cdot \delta\left(p+\delta(\delta v)-\frac{\delta u}{v}\right)_{k}=0
$$

summing by parts, we therefore obtain

$$
\begin{aligned}
& \frac{d}{d t}\left(\frac{1}{2} \sum u_{k}^{2} h\right)+\frac{d}{d t}\left(\frac{1}{2} \sum \delta v_{k}^{2} h\right)+\sum \frac{\delta u_{j}^{2}}{v_{j}} h-\sum p_{j} \delta u_{j} h=0 \\
& \quad \Rightarrow \int_{0}^{t} \sum p_{j} \delta u_{j} h=\int_{0}^{t}\left(\frac{d}{d s}\left(\frac{1}{2} \sum u_{k}^{2} h\right)+\frac{d}{d s}\left(\frac{1}{2} \sum \delta v_{k}^{2} h\right)+\sum \frac{\delta u_{j}^{2}}{v_{j}} h\right)
\end{aligned}
$$

then applying the Lemma 2.1 to obtain

$$
\left|\int_{0}^{t} \sum p_{j} \delta u_{j} h\right| \leq C
$$

since $p^{\prime}(v) v=C p(v)$ and $\dot{v}_{j}=\delta u_{j}$,

$$
\begin{aligned}
\left|\sum p_{j} v_{j} h\right| & =\left|\sum p_{j}(0) v_{j}(0) h+\int_{0}^{t} \sum \frac{d}{d s}\left(p_{j} v_{j}\right) h\right| \\
& =\left|\sum p_{j}(0) v_{j}(0) h+\int_{0}^{t} \sum p_{j} \dot{v}_{j} h+\int_{0}^{t} \sum \dot{p}_{j} v_{j} h\right| \\
& \leq C+\left|\int_{0}^{t} \sum p_{j}^{\prime} v_{j} \dot{v}_{j} h\right| \\
& \leq C+C\left|\int_{0}^{t} \sum p_{j} \delta u_{j} h\right| \\
& \leq C .
\end{aligned}
$$


In the above process, as a byproduct we obtain: $\left|I_{l}(t)\right| \leq C(t)$. Let's finish the proof of Lemma 2.2. By (2.10), we get

$$
v_{l}(t)=\frac{v_{l}(0)+I_{l}(t)}{v_{j^{\prime}(j, t)}(0) B_{l}(t) A(t)}
$$

combine with $0<C^{-1}(h) \leq v_{j} \leq C(h)$, uniformly for all $t$ and $j$, and (2.12), $(2.13)$

$$
v_{l}(t)=\frac{v_{l}(0)+I_{l}(t)}{v_{j^{\prime}(j, t)}(0) B_{l}(t) A(t)} \geq C(t)>0
$$

this proved (2.5).

Lemma 2.3. There is a positive constant $C(T)$ depending on $C_{0}$ and $T$ but independent of $h$ such that

$$
\begin{gathered}
\int_{0}^{T} \sum \delta(\delta v)_{j}^{2} h \leq C(T) \\
\sup _{0 \leq t \leq T}\left(t \sum \delta u_{j}^{2} h+t \sum \delta(\delta v)_{j}^{2} h\right)+\int_{0}^{T} t \sum \delta(\delta u)_{k}^{2} h \leq C(T) \\
\sup _{0 \leq t \leq T}\left(t^{2} \sum \dot{u}_{k}^{2} h+t^{2} \sum \delta \dot{v}_{k}^{2} h\right)+\int_{0}^{T} t^{2} \sum \delta \dot{u}_{j}^{2} h \leq C(T) \\
\sup _{0 \leq t \leq T}\left(t^{2} \sum \delta(\delta(\delta v))_{k}^{2} h\right) \leq C(T) \\
\sup _{0 \leq t \leq T}\left(t^{4} \sum \delta \dot{u}_{j}^{2} h+t^{4} \sum \delta(\delta \dot{v})_{j}^{2} h\right) \leq C(T) .
\end{gathered}
$$

Proof. Multiplying (2.2) by $\delta v_{k} h$, summing over $k$, integrating with respect to $t$, and rearranging, we arrive at

$$
\begin{gathered}
\int_{0}^{t} \sum \dot{u}_{k}(s) \delta v_{k}(s) h d s+\int_{0}^{t} \sum \delta p_{k} \delta v_{k} h d s \\
=\int_{0}^{t} \sum \delta\left(\frac{\delta u}{v}\right)_{k} \delta v_{k} h d s-\int_{0}^{t} \sum \delta(\delta(\delta v))_{k} \delta v_{k} h d s \\
\left.\sum u_{k} \delta v_{k}(s) h\right|_{0} ^{t}-\int_{0}^{t} \sum u_{k} \delta \dot{v}_{k} h+\int_{0}^{t} \sum \delta p_{k} \delta v_{k} h \\
=-\int_{0}^{t} \sum\left(\frac{\delta u}{v}\right)_{j} \delta(\delta v)_{j} h+\int_{0}^{t} \sum \delta(\delta v)_{j}^{2} h \\
\int_{0}^{t} \sum \delta(\delta v)_{j}^{2} h=\int_{0}^{t} \sum \frac{\delta u_{j} \delta(\delta v)_{j}}{v_{j}} h+\left.\sum u_{k} \delta v_{k}(s) h\right|_{0} ^{t} \\
\quad+\int_{0}^{t} \sum \delta u_{j}^{2} h+\int_{0}^{t} \sum \delta p_{k} \delta v_{k} h \\
\leq \varepsilon \int_{0}^{t} \sum \delta(\delta v)_{j}^{2} h+C(\varepsilon) \int_{0}^{t} \sum \frac{\delta u_{j}^{2}}{v_{j}} h+\sum u_{k}^{2}(t) h+\sum \delta v_{k}^{2}(t) h \\
+\int_{0}^{t} \sum \delta u_{j}^{2} h+C \int_{0}^{t} \sum \delta v_{k}^{2} h \leq C(T)
\end{gathered}
$$

this proved (2.17). 
In order to prove (2.18), we multiply both sides of $(2.2)$ by $s \delta(\delta u)_{k} h$, sum over $k$, and integrate with respect to $s$ on $[0, t]$. Summing by parts wherever necessary and rearranging terms, we obtain that

$$
\begin{aligned}
& \int_{0}^{t} s \sum \dot{u}_{k}(s) \delta(\delta u)_{k}(s) h d s+\int_{0}^{t} s \sum \delta p_{k} \delta(\delta u)_{k} h d s \\
& =\int_{0}^{t} s \sum \delta\left(\frac{\delta u}{v}\right)_{k} \delta(\delta u)_{k} h d s-\int_{0}^{t} s \sum \delta(\delta(\delta v))_{k} \delta(\delta u)_{k} h d s \\
& -\int_{0}^{t} s \sum \delta \dot{u}_{j} \delta u_{j} h+\int_{0}^{t} s \sum \delta p_{k} \delta(\delta u)_{k} h \\
& =\int_{0}^{t} s \sum\left(\frac{\delta u_{k+1 / 2}}{v_{k+1 / 2}}-\frac{\delta u_{k-1 / 2}}{v_{k-1 / 2}}\right)_{k} \delta(\delta u)_{k}-\int_{0}^{t} s \sum \delta(\delta(\delta v))_{k} \delta \dot{v}_{k} h \\
& -\int_{0}^{t} s \frac{d}{d s}\left(\frac{1}{2} \sum \delta u_{j}^{2} h\right)+\int_{0}^{t} s \sum \delta p_{k} \delta(\delta u)_{k} h \\
& =\int_{0}^{t} s \sum \frac{\delta u_{k+1 / 2} v_{k-1 / 2}-\delta u_{k-1 / 2} v_{k+1 / 2}}{v_{k+1 / 2} v_{k-1 / 2}} \delta(\delta u)_{k}+\int_{0}^{t} s \frac{d}{d s}\left(\frac{1}{2} \sum \delta(\delta v)_{j}^{2} h\right) \\
& -\int_{0}^{t} s \frac{d}{d s}\left(\frac{1}{2} \sum \delta u_{j}^{2} h\right)+\int_{0}^{t} s \sum \delta p_{k} \delta(\delta u)_{k} h \\
& =\int_{0}^{t} s \sum \frac{\delta(\delta u)_{k}^{2}}{v_{k+1 / 2}} h-\int_{0}^{t} s \sum \frac{\delta u_{k-1 / 2} \delta v_{k} \delta(\delta u)_{k}}{v_{k+1 / 2} v_{k-1 / 2}} h+\int_{0}^{t} s \frac{d}{d s}\left(\frac{1}{2} \sum \delta(\delta v)_{j}^{2} h\right) \\
& \frac{1}{2} t \sum \delta u_{j}^{2} h+\frac{1}{2} t \sum \delta(\delta v)_{j}^{2} h+\int_{0}^{t} s \sum \frac{\delta(\delta u)_{k}^{2}}{v_{k+1 / 2}} h \\
& =\int_{0}^{t} \frac{1}{2} \sum \delta u_{j}^{2} h+\int_{0}^{t} \frac{1}{2} \sum \delta(\delta v)_{j}^{2} h+\int_{0}^{t} s \sum \delta p_{k} \delta(\delta u)_{k} h \\
& +\int_{0}^{t} s \sum \frac{\delta u_{k-1 / 2} \delta v_{k} \delta(\delta u)_{k}}{v_{k+1 / 2} v_{k-1 / 2}} h \\
& \leq C(T)+\varepsilon \int_{0}^{t} s \sum \delta(\delta u)_{k}^{2} h+C(\varepsilon) \int_{0}^{t} s \sum \delta p_{k}^{2} h+\varepsilon \int_{0}^{t} s \sum \frac{\delta(\delta u)_{k}^{2}}{v_{k+1 / 2} v_{k-1 / 2}} h \\
& +C(\varepsilon) \int_{0}^{t} s \sum \frac{\delta u_{k-1 / 2}^{2} \delta v_{k}^{2}}{v_{k+1 / 2} v_{k-1 / 2}} h
\end{aligned}
$$

which leads to

$$
\begin{aligned}
& \sup _{0 \leq t \leq T}\left(t \sum \delta u_{j}^{2} h+t \sum \delta(\delta v)_{j}^{2} h\right)+\int_{0}^{T} t \sum \delta(\delta u)_{k}^{2} h \leq C(T) \\
& +C(T) \int_{0}^{T} t \sum \delta u_{k-1 / 2}^{2} \delta v_{k}^{2} h \leq C(T)+C(T) \sup _{0 \leq t \leq T}\left(t \max _{k} \delta v_{k}^{2}\right)
\end{aligned}
$$


we estimate $t \max _{k} \delta v_{k}^{2}$ as following

$$
\begin{aligned}
t \max _{k} \delta v_{k}^{2} & =t \delta v_{k_{0}}^{2}=t \sum_{0}^{k_{0}}\left(\delta v_{k}^{2}-\delta v_{k-1}^{2}\right)=t \sum_{0}^{k_{0}}\left(\delta v_{k}+\delta v_{k-1}\right) \delta(\delta v)_{j} h \\
& \leq t^{1 / 2}\left(\sum_{0}^{k_{0}}\left(\delta v_{k}+\delta v_{k-1}\right)^{2} h\right)^{1 / 2}\left(t \sum_{0}^{k_{0}} \delta(\delta v)_{j}^{2} h\right)^{1 / 2} \\
& \leq C(T)
\end{aligned}
$$

take the last inequality to (2.21), and apply the Young inequality with $\varepsilon$, we obtain (2.18).

We now multiply both sides of (2.2) by $s \dot{u}_{k} h$, sum by parts, and integrate with respect to $t$ to arrive at

$$
\begin{aligned}
& \int_{0}^{t} s \sum \dot{u}_{k}^{2} h d s+\int_{0}^{t} s \sum \delta p_{k} \dot{u}_{k} h d s \\
& =\int_{0}^{t} s \sum \delta\left(\frac{\delta u}{v}\right)_{k} \dot{u}_{k} h d s-\int_{0}^{t} s \sum \delta(\delta(\delta v))_{k} \dot{u}_{k} h d s \\
& \int_{0}^{t} s \sum \dot{u}_{k}^{2} h=-\int_{0}^{t} s \sum \delta p_{k} \dot{u}_{k} h+\int_{0}^{t} s \sum \delta\left(\frac{\delta u}{v}\right)_{k} \dot{u}_{k} h \\
& -\int_{0}^{t} s \sum \delta(\delta(\delta v))_{k} \dot{u}_{k} h \\
& =-\int_{0}^{t} s \sum \delta p_{k} \dot{u}_{k} h-\int_{0}^{t} s \sum \frac{\delta u_{j} \delta \dot{u}_{j}}{v} h+\int_{0}^{t} s \sum \delta(\delta v)_{j} \delta \dot{u}_{j} h \\
& \leq\left|\int_{0}^{t} s \sum \delta p_{k} \dot{u}_{k} h\right|+\left|\int_{0}^{t} s \sum \frac{\delta u_{j} \delta \dot{u}_{j}}{v} h\right|+\left|\int_{0}^{t} s \sum \delta(\delta v)_{j} \delta \dot{u}_{j} h\right| \\
& =: J_{1}+J_{2}+J_{3} \\
& J_{1}=\left|\int_{0}^{t} s \sum \delta p_{k} \dot{u}_{k} h\right| \leq \varepsilon \int_{0}^{t} s \sum \dot{u}_{k}^{2} h+C(\varepsilon) \int_{0}^{t} s \sum \delta p_{k}^{2} h \\
& \leq \varepsilon \int_{0}^{t} s \sum \dot{u}_{k}^{2} h+C(\varepsilon) \int_{0}^{t} s \sum\left(p^{\prime}(\xi) \delta v_{k}\right)^{2} h \quad \text { where } \xi \in\left[v_{k-1 / 2}, v_{k+1 / 2}\right] \\
& \leq \varepsilon \int_{0}^{t} s \sum \dot{u}_{k}^{2} h+C(\varepsilon) \cdot P \cdot \int_{0}^{t} s \sum \delta v_{k}^{2} h \\
& \leq \varepsilon \int_{0}^{t} s \sum \dot{u}_{k}^{2} h+C(T)
\end{aligned}
$$

where $P=\max \left(p\left(v_{j}\right), p^{\prime}\left(v_{j}\right), p^{\prime \prime}\left(v_{j}\right)\right)$ is arrived by $(2.5)$. 


$$
\begin{aligned}
J_{2} & =\left|\int_{0}^{t} s \sum \frac{\delta u_{j} \delta \dot{u}_{j}}{v} h\right|=\left|t \sum \frac{\delta u_{j}^{2}}{v_{j}} h-\int_{0}^{t} \frac{d}{d s}\left(\sum \frac{\delta u_{j}}{v_{j}}\right) \delta u_{j} h\right| \\
& =\left|\frac{1}{2} t \sum \frac{\delta u_{j}^{2}}{v_{j}} h-\frac{1}{2} \int_{0}^{t} \sum \frac{\delta u_{j}^{2}}{v_{j}} h+\frac{1}{2} \int_{0}^{t} s \sum \frac{\delta u_{j}^{3}}{v_{j}} h\right| \\
& \leq C(T)+C(T) \int_{0}^{t} s \sum\left|\delta u_{j}^{3}\right| h \\
J_{3} & =\left|\int_{0}^{t} s \sum \delta(\delta v)_{j} \delta \dot{u}_{j} h\right|=\left|\int_{0}^{t} s \sum \delta v_{k} \delta(\delta \dot{u})_{k} h\right| \\
& =\left|t \sum \delta v_{k} \delta(\delta u)_{k} h-\int_{0}^{t} \frac{d}{d s}\left(s \sum \delta v_{k}\right) \delta(\delta u)_{k} h\right| \\
& =\left|-t \sum \delta(\delta v)_{j} \delta u_{j} h+\int_{0}^{t} \sum \delta(\delta v)_{j} \delta u_{j} h-\int_{0}^{t} s \sum \delta(\delta u)_{k}^{2} h\right| \\
& \leq t \sum \delta(\delta v)_{j}^{2} h+t \sum \delta u_{j}^{2} h+\left|\int_{0}^{t} \sum \delta(\delta v)_{j} \dot{v}_{j} h\right|+\left|\int_{0}^{t} s \sum \delta(\delta u)_{k}^{2} h\right| \\
& \leq C(T)+\left|\int_{0}^{t} \sum \delta(\delta v)_{j} \dot{v}_{j} h\right| \\
& \leq C(T)+\sum \delta v_{k}^{2} h \\
& \leq C(T)
\end{aligned}
$$

substituting $J_{1}, J_{2}, J_{3}$ into $(2.23)$

$$
\begin{aligned}
\int_{0}^{t} s \sum \dot{u}_{k}^{2} h \leq & C(T)+C(T) \int_{0}^{t} s \sum\left|\delta u_{j}^{3}\right| h \leq C(T) \\
& +C(T) \sup _{0 \leq t \leq T}\left(t \max \left|\delta u_{j}\right|\right)
\end{aligned}
$$

Next, by differentiating (2.2) with respect to $s$, multiplying by $s^{2} \dot{u}_{k} h$, summing over $k$, and integrating, we show that

$$
\begin{aligned}
& \int_{0}^{t} s^{2} \sum \ddot{u}_{k} \dot{u}_{k} h d s+\int_{0}^{t} s^{2} \sum \delta \dot{p}_{k} \dot{u}_{k} h d s \\
&=\int_{0}^{t} s^{2} \sum \frac{d}{d s}\left(\delta\left(\frac{\delta u}{v}\right)_{k}\right) \dot{u}_{k} h d s-\int_{0}^{t} s^{2} \sum \delta(\delta(\delta \dot{v}))_{k} \dot{u}_{k} h d s \\
& \frac{1}{2} \int_{0}^{t} s^{2} \frac{d}{d s}\left(\sum \dot{u}_{k}^{2} h\right)+\frac{1}{2} \int_{0}^{t} s^{2} \frac{d}{d s}\left(\sum \delta \dot{v}_{k}^{2} h\right) \\
&=-\int_{0}^{t} s^{2} \sum \frac{d}{d s}\left(\frac{\delta u}{v}\right)_{j} \delta \dot{u}_{j} h d s-\int_{0}^{t} s^{2} \sum \delta \dot{p}_{k} \dot{u}_{k} h \\
&=-\int_{0}^{t} s^{2} \sum \frac{\delta \dot{u}_{j} v_{j}-\delta u_{j} \dot{v}_{j}}{v_{j}^{2}} \delta \dot{u}_{j} h+\int_{0}^{t} s^{2} \sum \dot{p}_{j} \delta \dot{u}_{j} h \\
&=-\int_{0}^{t} s^{2} \sum \frac{\delta \dot{u}_{j}^{2}}{v_{j}} h+\int_{0}^{t} s^{2} \sum \frac{\delta u_{j}^{2} \delta \dot{u}_{j}}{v_{j}^{2}} h+\int_{0}^{t} s^{2} \sum \dot{p}_{j} \delta \dot{u}_{j} h
\end{aligned}
$$




$$
\begin{aligned}
& \frac{1}{2} t^{2} \sum \dot{u}_{k}^{2} h+\frac{1}{2} t^{2} \sum \delta \dot{v}_{k}^{2} h-\int_{0}^{t} s \sum \dot{u}_{k}^{2} h-\int_{0}^{t} s \sum \delta \dot{v}_{k}^{2} h \\
& =-\int_{0}^{t} s^{2} \sum \frac{\delta \dot{u}_{j}^{2}}{v_{j}} h+\int_{0}^{t} s^{2} \sum \frac{\delta u_{j}^{2} \delta \dot{u}_{j}}{v_{j}^{2}} h+\int_{0}^{t} s^{2} \sum \dot{p}_{j} \delta \dot{u}_{j} h \\
& \frac{1}{2} t^{2} \sum \dot{u}_{k}^{2} h+\frac{1}{2} t^{2} \sum \delta \dot{v}_{k}^{2} h+\int_{0}^{t} s^{2} \sum \frac{\delta \dot{u}_{j}^{2}}{v_{j}} h \\
& =\int_{0}^{t} s \sum \dot{u}_{k}^{2} h+\int_{0}^{t} s \sum \delta \dot{v}_{k}^{2} h+\int_{0}^{t} s^{2} \sum \frac{\delta u_{j}^{2} \delta \dot{u}_{j}}{v_{j}^{2}} h+\int_{0}^{t} s^{2} \sum \dot{p}_{j} \delta \dot{u}_{j} h \\
& =: \int_{0}^{t} s \sum \dot{u}_{k}^{2} h+K_{1}+K_{2}+K_{3} \\
& K_{1}=\int_{0}^{t} s \sum \delta \dot{v}_{k}^{2} h=\int_{0}^{t} s \sum \delta \dot{v}_{k} \delta(\delta u)_{k} h \leq \varepsilon \int_{0}^{t} s \sum \delta \dot{v}_{k}^{2} h+C(\varepsilon) \int_{0}^{t} s \sum \delta(\delta u)_{k}^{2} h \\
& K_{2}=\int_{0}^{t} s^{2} \sum \frac{\delta u_{j}^{2} \delta \dot{u}_{j}}{v_{j}^{2}} h \leq \varepsilon \int_{0}^{t} s^{2} \sum \delta \dot{u}_{j}^{2} h+C(\varepsilon) \int_{0}^{t} s^{2} \sum \delta u_{j}^{4} h \\
& K_{3}=\int_{0}^{t} s^{2} \sum \dot{p}_{j} \delta \dot{u}_{j} h \leq \varepsilon \int_{0}^{t} s^{2} \sum \delta \dot{u}_{j}^{2} h+C(\varepsilon) \int_{0}^{t} s^{2} \sum \dot{p}_{j}^{2} h \\
& \leq \varepsilon \int_{0}^{t} s^{2} \sum \delta \dot{u}_{j}^{2} h+C(\varepsilon) \cdot P \cdot \int_{0}^{t} s^{2} \sum \dot{v}_{j}^{2} h \\
& \leq \varepsilon \int_{0}^{t} s^{2} \sum \delta \dot{u}_{j}^{2} h+C(\varepsilon) \int_{0}^{t} s^{2} \sum \delta u_{j}^{2} h \leq \varepsilon \int_{0}^{t} s^{2} \sum \delta \dot{u}_{j}^{2} h+C(T)
\end{aligned}
$$

then we get

$$
\begin{aligned}
& \sup _{0 \leq t \leq T}\left(t^{2} \sum \dot{u}_{k}^{2} h+t^{2} \sum \delta \dot{v}_{k}^{2} h\right)+\int_{0}^{T} t^{2} \sum \delta \dot{u}_{j}^{2} h \\
& \leq C(T)+C(T) \int_{0}^{T} t \sum \dot{u}_{k}^{2} h+C(T) \int_{0}^{T} t^{2} \sum \delta u_{j}^{4} h
\end{aligned}
$$

substituting (2.24) into (2.25), we then obtain

$$
\begin{aligned}
& \sup _{0 \leq t \leq T}\left(t^{2} \sum \dot{u}_{k}^{2} h+t^{2} \sum \delta \dot{v}_{k}^{2} h\right)+\int_{0}^{T} t^{2} \sum \delta \dot{u}_{j}^{2} h \leq C(T) \\
& \quad+C(T) \sup _{0 \leq t \leq T}\left(t^{2} \max _{j}\left|\delta u_{j}\right|^{2}\right)
\end{aligned}
$$

Dealing with $t^{2} \max _{j}\left|\delta u_{j}\right|^{2}$ similar to $t \max _{j}\left|\delta v_{k}\right|^{2}$ :

$$
\begin{aligned}
t^{2} \max _{j}\left|\delta u_{j}\right|^{2} & \leq C t^{2}\left(\sum \delta u_{j}^{2} h\right)^{1 / 2}\left(\sum \delta(\delta u)_{k}^{2} h\right)^{1 / 2} \\
& \leq C\left(t^{2} \sum \delta u_{j}^{2} h+t^{2} \sum \delta \dot{v}_{k}^{2} h\right) \leq C(T)
\end{aligned}
$$

inserting this inequality into (2.26), we obtain (2.19). 
From Eq. (2.2),

$$
\begin{aligned}
t^{2} \sum \delta(\delta(\delta v))_{k}^{2} h & =t^{2} \sum\left(\frac{\delta(\delta u)_{k}}{v_{k+1 / 2}}-\frac{\delta u_{k-1 / 2} \delta v_{k}}{v_{k+1 / 2} v_{k-1 / 2}}-\dot{u}_{k}-p^{\prime}(\xi) \delta v_{k}\right)^{2} h \\
& \leq C(T)\left(t^{2} \sum \dot{u}_{k}^{2} h+t^{2} \sum \delta v_{k}^{2} h+t^{2} \sum \delta(\delta u)_{k}^{2} h+t^{2} \sum \delta u_{j}^{2} \delta v_{k}^{2} h\right) \\
& \leq C(T)\left(1+t^{2} \max _{j}\left|\delta u_{j}\right|^{2} \sum \delta v_{k}^{2} h\right) \\
& \leq C(T) .
\end{aligned}
$$

this proved (2.20).

In order to prove (2.21), we first differentiate (2.2) with respect to $s$, multiply by $s^{3} \delta \dot{v}_{k} h$, sum by parts, integrate with respect to $s$ to arrive at

$$
\begin{aligned}
& \int_{0}^{t} s^{3} \sum \ddot{u}_{k} \delta \dot{v}_{k} h+\int_{0}^{t} s^{3} \sum \delta \dot{p}_{k} \delta \dot{v}_{k} h \\
& =\int_{0}^{t} s^{3} \sum \frac{d}{d s}\left(\delta\left(\frac{\delta u}{v}\right)_{k}\right) \delta \dot{v}_{k} h-\int_{0}^{t} s^{3} \sum \delta(\delta(\delta \dot{v}))_{k} \delta \dot{v}_{k} h \\
& \Rightarrow \int_{0}^{t} s^{3} \sum \delta(\delta \dot{v})_{j}^{2} h=\int_{0}^{t} s^{3} \sum \ddot{u}_{k} \delta \dot{v}_{k} h+\int_{0}^{t} s^{3} \sum \delta \dot{p}_{k} \delta \dot{v}_{k} h \\
& +\int_{0}^{t} s^{3} \sum \frac{d}{d s}\left(\frac{\delta u}{v}\right)_{j} \delta(\delta \dot{v})_{j} h \\
& \int_{0}^{t} s^{3} \sum \ddot{u}_{k} \delta \dot{v}_{k} h=t^{3} \sum \dot{u}_{k} \delta \dot{v}_{k} h-3 \int_{0}^{t} s^{2} \sum \dot{u}_{k} \delta \dot{v}_{k} h-\int_{0}^{t} s^{3} \sum \dot{u}_{k} \delta \ddot{v}_{k} h \\
& \leq t^{3} \sum \dot{u}_{k}^{2} h+t^{3} \sum \delta \dot{v}_{k}^{2} h+3 \int_{0}^{t} s^{2} \sum \dot{u}_{k}^{2} h+3 \int_{0}^{t} s^{2} \sum \delta \dot{v}_{k}^{2} h \\
& +\int_{0}^{t} s^{3} \sum \delta \dot{u}_{j}^{2} h \\
& \leq C(T) \\
& \int_{0}^{t} s^{3} \sum \delta \dot{p}_{k} \delta \dot{v}_{k} h \leq\left(1+P^{2}\right) \int_{0}^{t} s^{3} \sum \delta \dot{v}_{k}^{2} h \leq C(T) \\
& \int_{0}^{t} s^{3} \sum \frac{d}{d s}\left(\frac{\delta u}{v}\right)_{j} \delta(\delta \dot{v})_{j} h \\
& \leq \varepsilon \int_{0}^{t} s^{3} \sum \delta(\delta \dot{v})_{j}^{2} h+C(\varepsilon) \int_{0}^{t} s^{3} \sum\left(\frac{\delta \dot{u}_{j}}{v_{j}}-\frac{\delta u_{j}^{2}}{v_{j}^{2}}\right)^{2} h \\
& \leq \varepsilon \int_{0}^{t} s^{3} \sum \delta(\delta \dot{v})_{j}^{2} h \\
& +C(T) \int_{0}^{t} s^{2} \sum \delta \dot{u}_{j}^{2} h+C(T) \sup _{0 \leq t \leq T}\left(t^{2} \max _{j}\left|\delta u_{j}\right|^{2}\right) \int_{0}^{t} \sum \delta u_{j}^{2} h \\
& \leq C(T)+\varepsilon \int_{0}^{t} s^{3} \sum \delta(\delta \dot{v})_{j}^{2} h
\end{aligned}
$$


insert the last three inequality into (2.27), we obtain

$$
\int_{0}^{t} s^{3} \sum \delta(\delta \dot{v})_{j}^{2} h \leq C(T)
$$

Differentiating (2.2) with respect to $s$, multiplying by $s^{4} \delta(\delta \dot{u})_{k} h$, integrating, and summing by parts, we can also obtain that

$$
\begin{aligned}
& \frac{1}{2} t^{4} \sum \delta \dot{u}_{j}^{2} h+\frac{1}{2} t^{4} \sum \delta(\delta \dot{v})_{j}^{2} h+\int_{0}^{t} s^{4} \sum \frac{d}{d s}\left(\delta\left(\frac{\delta u}{v}\right)_{k}\right) \delta(\delta \dot{u})_{k} h \\
& \quad=2 \int_{0}^{t} s^{3} \sum \delta \dot{u}_{j}^{2} h+2 \int_{0}^{t} t^{3} \sum \delta(\delta \dot{v})_{j}^{2} h+\int_{0}^{t} s^{4} \sum \delta \dot{p}_{k} \delta(\delta \dot{u})_{k} h \\
& \leq C(T)+\int_{0}^{t} s^{4} \sum \delta \dot{p}_{k} \delta(\delta \dot{u})_{k} h \\
& \int_{0}^{t} s^{4} \sum \delta \dot{p}_{k} \delta(\delta \dot{u})_{k} h=-\int_{0}^{t} s^{4} \sum \dot{p}_{j} \delta(\delta \ddot{v})_{j} h=-\int_{0}^{t} s^{4} \sum p^{\prime}(\xi) \dot{v}_{j} \delta(\delta \ddot{v})_{j} h \\
& \leq C\left|\int_{0}^{t} s^{4} \sum \dot{v}_{j} \delta(\delta \ddot{v})_{j} h\right| \leq C\left|\int_{0}^{t} s^{4} \sum \delta \dot{v}_{k} \delta \ddot{v}_{k} h\right| \\
& \leq C\left|\int_{0}^{t} s^{4} \sum \frac{d}{d s}\left(\delta \dot{v}_{k}^{2}\right) h\right| \\
& \leq C t^{4} \sum \delta \dot{v}_{k}^{2} h+\leq C \int_{0}^{t} s^{3} \sum \delta \dot{v}_{k}^{2} h \\
& \leq C(T)
\end{aligned}
$$

insert this into the above inequality to obtain

$$
\begin{aligned}
& \frac{1}{2} t^{4} \sum \delta \dot{u}_{j}^{2} h+\frac{1}{2} t^{4} \sum \delta(\delta \dot{v})_{j}^{2} h+\int_{0}^{t} s^{4} \sum \frac{d}{d s}\left(\delta\left(\frac{\delta u}{v}\right)_{k}\right) \delta(\delta \dot{u})_{k} h \leq C(T) \\
& \frac{1}{2} t^{4} \sum \delta \dot{u}_{j}^{2} h+\frac{1}{2} t^{4} \sum \delta(\delta \dot{v})_{j}^{2} h \\
& \quad+\int_{0}^{t} s^{4} \sum \frac{d}{d s}\left(\frac{\delta(\delta u)_{k}}{v_{k+1 / 2}}-\frac{\delta u_{k-1 / 2} \delta v_{k}}{v_{k+1 / 2} v_{k-1 / 2}}\right) \delta(\delta \dot{u})_{k} h \leq C(T) \\
& \frac{1}{2} t^{4} \sum \delta \dot{u}_{j}^{2} h+\frac{1}{2} t^{4} \sum \delta(\delta \dot{v})_{j}^{2} h+\int_{0}^{t} s^{4} \sum \frac{\delta(\delta \dot{u})_{k}^{2}}{v_{k+1 / 2}} h \\
& \leq C(T)+\int_{0}^{t} s^{4} \sum \frac{\delta(\delta \dot{u})_{k}}{v_{k+1 / 2} v_{k-1 / 2}}\left(\frac{d}{d s}\left(\delta u_{k-1 / 2} \delta v_{k}\right)\right) h \\
& \quad+\int_{0}^{t} s^{4} \sum \frac{\delta(\delta u)_{k} \delta u_{k+1 / 2}}{v_{k+1 / 2}^{2}} \delta(\delta \dot{u})_{k} h \\
& \quad-\int_{0}^{t} s^{4} \sum \frac{\delta(\delta \dot{u})_{k}\left(\delta u_{k-1 / 2} \delta v_{k}\right)}{\left(v_{k+1 / 2} v_{k-1 / 2}\right)^{2}} \frac{d}{d s}\left(v_{k+1 / 2} v_{k-1 / 2}\right) h \\
& =: C(T)+L_{1}+L_{2}-L_{3}
\end{aligned}
$$




$$
\begin{aligned}
& \left|-L_{3}\right|=\left|-\int_{0}^{t} s^{4} \sum \frac{\delta(\delta \dot{u})_{k}\left(\delta u_{k-1 / 2} \delta v_{k}\right)}{\left(v_{k+1 / 2} v_{k-1 / 2}\right)^{2}} \frac{d}{d s}\left(v_{k+1 / 2} v_{k-1 / 2}\right) h\right| \\
& \leq C(T)\left|\int_{0}^{t} s^{4} \sum \delta v_{k} \delta(\delta \dot{u})_{k} h\right| \\
& \leq C(T)\left|\int_{0}^{t} s^{4} \sum \delta(\delta v)_{j} \delta \dot{u}_{j} h\right| \\
& \leq C(T) \int_{0}^{t} s^{4} \sum \delta(\delta v)_{j}^{2} h+C(T) \int_{0}^{t} s^{4} \sum \delta \dot{u}_{j}^{2} h \\
& \leq C(T) \\
& \left|L_{2}\right|=\left|\int_{0}^{t} s^{4} \sum \frac{\delta(\delta u)_{k} \delta u_{k+1 / 2}}{v_{k+1 / 2}^{2}} \delta(\delta \dot{u})_{k} h\right| \leq C(T)\left|\int_{0}^{t} s^{4} \sum \delta(\delta u)_{k} \delta(\delta \dot{u})_{k} h\right| \\
& \leq C(T) t^{4} \sum \delta(\delta u)_{k}^{2} h+\int_{0}^{t} s^{3} \sum \delta(\delta u)_{k}^{2} h \leq C(T) \\
& \left|L_{1}\right|=\left|\int_{0}^{t} s^{4} \sum \frac{\delta(\delta \dot{u})_{k}}{v_{k+1 / 2} v_{k-1 / 2}}\left(\frac{d}{d s}\left(\delta u_{k-1 / 2} \delta v_{k}\right)\right) h\right| \\
& =\left|\int_{0}^{t} s^{4} \sum \frac{\delta(\delta \dot{u})_{k}}{v_{k+1 / 2} v_{k-1 / 2}}\left(\left(\delta \dot{u}_{k-1 / 2} \delta v_{k}\right)+\left(\delta u_{k-1 / 2} \delta \dot{v}_{k}\right)\right) h\right| \\
& \leq C(T)\left|\int_{0}^{t} s^{3} \sum \frac{\delta \dot{u}_{k-1 / 2} \delta(\delta \dot{u})_{k}}{v_{k+1 / 2} v_{k-1 / 2}} h\right|+\left|\int_{0}^{t} s^{4} \sum \frac{\delta(\delta \dot{u})_{k}\left(\delta u_{k-1 / 2} \delta \dot{v}_{k}\right)}{v_{k+1 / 2} v_{k-1 / 2}} h\right| \\
& \leq C(T, \varepsilon) \int_{0}^{t} s^{2} \sum \delta \dot{u}_{j}^{2} h+\varepsilon \int_{0}^{t} s^{4} \sum \frac{\delta(\delta \dot{u})_{k}^{2}}{v_{k+1 / 2}} h+\varepsilon \int_{0}^{t} s^{4} \sum \frac{\delta(\delta \dot{u})_{k}^{2}}{v_{k+1 / 2}} h \\
& +C(T, \varepsilon) \sup _{0 \leq t \leq T}\left(t^{2} \max _{j}\left|\delta u_{j}\right|^{2}\right) \int_{0}^{t} s^{2} \sum \delta \dot{v}_{k}^{2} h \\
& \leq C(T)+\varepsilon \int_{0}^{t} s^{4} \sum \frac{\delta(\delta \dot{u})_{k}^{2}}{v_{k+1 / 2}} h
\end{aligned}
$$

and then we get

$$
\frac{1}{2} t^{4} \sum \delta \dot{u}_{j}^{2} h+\frac{1}{2} t^{4} \sum \delta(\delta \dot{v})_{j}^{2} h+\int_{0}^{t} s^{4} \sum \frac{\delta(\delta \dot{u})_{k}^{2}}{v_{k+1 / 2}} h \leq C(T)
$$

this proved (2.21), and we now complete the proof of Lemma 2.3.

\section{Weak solution}

We construct approximate solutions $v^{h}, u^{h}$ as follows: $v^{h}\left(x_{j}, t\right)=v_{j}(t)$ for $j+\frac{1}{2} \in \mathbb{Z}_{+}$, and $u^{h}\left(x_{k}, t\right)=u_{k}(t)$ for $k \in \mathbb{Z}_{+} \cup\{0\}$, and take $v^{h}(\cdot, t)$ and $u^{h}(\cdot, t)$ to be linear on each interval $\left[x_{j}, x_{j+1}\right]$ and $\left[x_{k}, x_{k+1}\right]$,respectively. Below we state a number of regularity properties of $v^{h}$ and $u^{h}$. 
Lemma 3.1. There are positive constants $C, C(T)$ depending on $C_{0}$ and $C_{0}$, $T$, but independent of $h$ such that

$$
\begin{aligned}
& 0<\frac{1}{C(T)} \leq v^{h}(t), 0 \leq t \leq T \\
& \sup _{0 \leq t \leq T}\left(\int_{0}^{\infty}\left(v^{h}(x, t)-v^{\prime}\right)^{2} d x+\int_{0}^{\infty} v_{x}^{h}(x, t)^{2} d x+t^{2} \int_{0}^{\infty} \dot{v}_{x}^{h}(x, t)^{2} d x\right) \\
& \quad \leq C(T) \\
& \sup _{0 \leq t \leq T}\left(\int_{0}^{\infty} u^{h}(x, t)^{2} d x+t \int_{0}^{\infty} u_{x}^{h}(x, t)^{2} d x\right) \leq C(T) \\
& \sup _{0 \leq t \leq T}\left(t^{2} \int_{0}^{\infty} \dot{u}^{h}(x, t)^{2} d x+t^{4} \int_{0}^{\infty} \dot{u}_{x}^{h}(x, t)^{2} d x\right) \leq C(T) \\
& \sup _{0 \leq t \leq T}\left(t \int_{0}^{\infty} \dot{v}^{h}(x, t)^{2} d x+t^{2} \int_{0}^{\infty} \dot{v}_{x}^{h}(x, t)^{2} d x\right) \leq C(T) \\
& \left\|v^{h}\left(\cdot, t_{2}\right)-v^{h}\left(\cdot, t_{1}\right)\right\|_{L^{2}} \leq C(T)\left|t_{2}-t_{1}\right|^{1 / 2} .
\end{aligned}
$$

There is also a positive constant $C(T, \tau)$ depending on $C_{0}, T$, and $\tau$, such that for any $0<\tau \leq t_{1}<t_{2} \leq T$

$$
\begin{aligned}
\left|u^{h}\left(x_{1}, t_{1}\right)-u^{h}\left(x_{2}, t_{2}\right)\right| & \leq C(T, \tau)\left(\left|x_{1}-x_{2}\right|^{1 / 2}+\left|t_{2}-t_{1}\right|\right) \\
\left|v^{h}\left(x_{1}, t_{1}\right)-v^{h}\left(x_{2}, t_{2}\right)\right| & \leq C(T, \tau)\left(\left|x_{1}-x_{2}\right|^{1 / 2}+\left|t_{2}-t_{1}\right|\right) \\
\left|u_{x}^{h}\left(x_{1}, t_{1}\right)-u_{x}^{h}\left(x_{2}, t_{2}\right)\right| & \leq C(T, \tau)\left(\left|x_{1}-x_{2}\right|^{1 / 2}+\left|t_{2}-t_{1}\right|^{1 / 4}+h^{1 / 2}\right) \\
\left|v_{x}^{h}\left(x_{1}, t_{1}\right)-v_{x}^{h}\left(x_{2}, t_{2}\right)\right| & \leq C(T, \tau)\left(\left|x_{1}-x_{2}\right|^{1 / 2}+\left|t_{2}-t_{1}\right|^{1 / 4}+h^{1 / 2}\right)
\end{aligned}
$$

Proof. Inequalities (3.1)-(3.5) follow immediately from (2.4), (2.5) and (2.17)(2.21). To prove (3.6) we refer to the inequality

$$
\begin{aligned}
\int_{0}^{\infty}\left(v^{h}\left(x, t_{2}\right)-v^{h}\left(x, t_{1}\right)\right)^{2} d x & \leq \int_{0}^{\infty}\left|\int_{t_{1}}^{t_{2}}\right| \dot{v}^{h}(x, t)|d t|^{2} d x \\
& \leq\left|t_{2}-t_{1}\right| \int_{t_{1}}^{t_{2}} \int_{0}^{\infty} u_{x}^{h}(x, t) d x d t \leq C(T)\left|t_{2}-t_{1}\right| .
\end{aligned}
$$

Next, $v^{h}$ and $u^{h}$ are Hölder continuous with respect to $x$ due to (3.2) and (3.3). Also,

$$
\begin{aligned}
\left|u^{h}\left(x, t_{1}\right)-u^{h}\left(x, t_{2}\right)\right| & \leq \int_{t_{1}}^{t_{2}}\left\|\dot{u}^{h}(\cdot, t)||_{L^{\infty}} d t \leq \int_{t_{1}}^{t_{2}}\right\| \dot{u}^{h}(\cdot, t)\left\|_{L^{2}}^{1 / 2}\right\| \dot{u}_{x}^{h}(\cdot, t) \|_{L^{2}}^{1 / 2} d t \\
& \leq C(T, \tau)\left|t_{2}-t_{1}\right|
\end{aligned}
$$

by (3.4). This completes the proof of (3.7), and the proof of (3.8) is similar.

The derivation of (3.9) is the most involved, and we will treat it by three steps below: 
Step one, when $x_{1}, x_{2}$ are two of the net pots of $x_{j}$ defined as in Sect 2 , we then have

$$
\begin{aligned}
\left|u_{x}^{h}\left(x_{j}, t\right)-u_{x}^{h}\left(x_{j^{\prime}}, t\right)\right| & \leq\left|\sum_{j<k<j^{\prime}} \delta(\delta u)_{k} h\right| \leq\left(\sum_{j<k<j^{\prime}} \delta(\delta u)_{k}^{2} h\right)^{1 / 2}\left(\sum_{j<k<j^{\prime}} h\right)^{1 / 2} \\
& \leq C(T, \tau)\left|x_{j}-x_{j^{\prime}}\right|^{1 / 2}
\end{aligned}
$$

follows directly from (2.19), where $\delta \dot{v}_{k}=\delta(\delta u)_{k}$.

Step two, now we derive time-regularity bound. Let $J$ be a large positive integer. Then by (3.11)

$$
\begin{aligned}
\left|u_{x}^{h}\left(x_{j}, t_{1}\right)-u_{x}^{h}\left(x_{j}, t_{2}\right)\right| \leq & \left|u_{x}^{h}\left(x_{j}, t_{1}\right)-u_{x}^{h}\left(x_{i}, t_{1}\right)\right|+\left|u_{x}^{h}\left(x_{i}, t_{1}\right)-u_{x}^{h}\left(x_{i}, t_{2}\right)\right| \\
& +\left|u_{x}^{h}\left(x_{j}, t_{2}\right)-u_{x}^{h}\left(x_{i}, t_{2}\right)\right| \\
\leq & \left|u_{x}^{h}\left(x_{i}, t_{1}\right)-u_{x}^{h}\left(x_{i}, t_{2}\right)\right|+C(T, \tau)\left|x_{j}-x_{j^{\prime}}\right|^{1 / 2} \Rightarrow \\
\left|u_{x}^{h}\left(x_{j}, t_{1}\right)-u_{x}^{h}\left(x_{j}, t_{2}\right)\right| \leq & \frac{1}{J h} \sum_{|i-j|<J}\left|u_{x}^{h}\left(x_{i}, t_{1}\right)-u_{x}^{h}\left(x_{i}, t_{2}\right)\right| h+C(T, \tau)(J h)^{1 / 2} \\
\leq & \sqrt{\frac{t_{2}-t_{1}}{J h}}\left(\int_{t_{1}}^{t_{2}} \dot{u}_{x}^{h}(\cdot, t)^{2} h d t\right)^{1 / 2}+C(T, \tau)(J h)^{1 / 2} \\
\leq & C(T, \tau)\left(\sqrt{\frac{t_{2}-t_{1}}{J h}}+(J h)^{1 / 2}\right) \\
\leq & C(T, \tau)\left(\left|t_{2}-t_{1}\right|^{1 / 4}+(J h)^{1 / 2}\right)
\end{aligned}
$$

we choose $J$ so that $J h-h<\sqrt{t_{2}-t_{1}} \leq J h$. So (3.9) holds for $x_{1}, x_{2}$ are two of the net pots of $x_{j}$ defined as in Sect. 2 , and arbitrary $t_{1}, t_{2}>0$.

Step three, when $x_{1} \in\left(x_{j}, x_{j+1}\right)$ then:

$$
u_{x}^{h}\left(x_{1}, t\right)=\delta u_{j}(t), \quad \text { when } x_{1} \in\left(x_{j}, x_{j+1 / 2}\right)
$$

or else

$$
u_{x}^{h}\left(x_{1}, t\right)=\delta u_{j+1}(t), \quad \text { when } x_{1} \in\left(x_{j+1 / 2}, x_{j+1}\right)
$$

and when $x_{1}=x_{j+1 / 2}$, we can define supplement by $u_{x}^{h}\left(x_{1}, t\right)=\delta u_{j}(t)$. Thus else

$$
\left|u_{x}^{h}\left(x_{1}, t\right)-u_{x}^{h}\left(x_{j}, t\right)\right|=0
$$

or else

$$
\left|u_{x}^{h}\left(x_{1}, t\right)-u_{x}^{h}\left(x_{j}, t\right)\right|=\left|\delta u_{j}-\delta u_{j+1}\right| \leq C(T, \tau) h^{1 / 2}
$$

this proved (3.9). And we have completed this Lemma.

Lemma 3.2. There is a sequence $h=\left\{h_{l}\right\} \rightarrow 0$ and functions $u,\left(v-v^{\prime}\right) \in$ $H^{1}([0, \infty))$, such that:

$$
\begin{aligned}
& u^{h}-u, u_{x}^{h}-u_{x}, v^{h}-v, v_{x}^{h}-v_{x} \rightarrow 0 \text { uniformly on compact sets in } \\
& {[0, \infty) \times(t>0) .}
\end{aligned}
$$

Proof. By the Hölder continuous and bounded estimate. It's a direct consequence of Lemma 3.1. 
Now we define a new variable $w_{*}^{h}$ by $w_{*}^{h}\left(x_{k}, t\right)=\delta v_{k}$ and taking $w_{*}^{h}$ to be linear on $\left[x_{k}, x_{k+1}\right], k=0,1, \ldots$ We also introduce $w^{h}$ :

$$
w^{h}(x, t)=v^{h}(0, t)+\int_{0}^{x} w_{x}^{h}(y, t) d y .
$$

Both variables are necessary to show that the limit of $v^{h}$ as $h \rightarrow 0$ satisfies high order regularity estimates. Below we list several properties of the new variables.

Lemma 3.3. There is a positive constant $C(T, \tau)$ depending on $C_{0}, T$, and $\tau$, such that for any $0<\tau \leq t_{1}<t_{2} \leq T$ :

$$
\begin{aligned}
& \sup _{0 \leq t \leq T}\left(t \int_{0}^{\infty} w_{* x}^{h}(x, t)^{2} d x+t^{4} \int_{0}^{\infty} \dot{w}_{* x}^{h}(x, t)^{2} d x+t^{2} \int_{0}^{\infty} w_{x x x}^{h}(x, t)^{2} d x\right) \\
& \quad \leq C(T), \\
& \left|w_{*}^{h}\left(x_{1}, t_{1}\right)-w_{*}^{h}\left(x_{2}, t_{2}\right)\right| \leq C(T, \tau)\left(\left|x_{1}-x_{2}\right|^{1 / 2}+\left|t_{2}-t_{1}\right|\right) \\
& \left|w_{* x}^{h}\left(x_{1}, t_{1}\right)-w_{* x}^{h}\left(x_{2}, t_{2}\right)\right| \leq C(T, \tau)\left(\left|x_{1}-x_{2}\right|^{1 / 2}+\left|t_{2}-t_{1}\right|^{1 / 4}+h^{1 / 2}\right)
\end{aligned}
$$

Proof. Inequality (3.14) follows from (2.18), (2.20) and (2.21), and the proof (3.15) and (3.16) is similar to those of (3.7)-(3.10).

Lemma 3.4. There is a sequence $h=\left\{h_{l}\right\} \rightarrow 0$ and functions $w_{*} \in H^{1}([0, \infty))$, $w \in H^{2}([0, \infty))$, such that:

$$
\begin{aligned}
& w_{* x}^{h}-w_{* x}, w^{h}-w, w_{x}^{h}-w_{x}, w_{x x}^{h}-w_{x x} \rightarrow 0 \text { uniformly } \\
& \text { on compact sets in }[0, \infty) \times(t>0) .
\end{aligned}
$$

Proof. It's a direct consequence of Lemma 3.3.

Lemma 3.5. $v \equiv w$.

Proof. We observe that $v^{h}\left(x_{k}, t\right)=w^{h}\left(x_{k}, t\right)$ for any $k$. Obviously $w^{h}(0, t)=$ $v^{h}(0, t)$, and

$$
\begin{aligned}
w^{h}\left(x_{k}\right)-w^{h}\left(x_{k-1}\right) & =\int_{x_{k-1}}^{x_{k}} w_{*}^{h}(y) d y=\frac{h}{2}\left(w_{*}^{h}\left(x_{k}\right)-w_{*}^{h}\left(x_{k-1}\right)\right) \\
& =\frac{1}{2}\left(v_{k+1 / 2}+v_{k-3 / 2}\right)
\end{aligned}
$$

and

$$
\begin{aligned}
v^{h}\left(x_{k}, t\right)-v^{h}\left(x_{k-1}, t\right) & =\frac{1}{2}\left(v_{k+1 / 2}+v_{k-1 / 2}\right)-\frac{1}{2}\left(v_{k-1 / 2}-v_{k-3 / 2}\right) \\
& =\frac{1}{2}\left(v_{k+1 / 2}+v_{k-3 / 2}\right)
\end{aligned}
$$

for all $k \geq 1$. We now recall the space regularity of $v^{h}(\cdot, t)$ and $w^{h}(\cdot, t)$ to complete the proof.

Finally we should derive that the limiting functions $v$ and $u$ are in fact the weak solution of (1.1)-(1.4). 
Proof of Theorem 1. (1.18) is a direct consequence of (3.1) and (3.12), and we can obtain (1.19) from (3.2) and (3.3), since weak convergence is normdecreasing.

Let $\phi$ be a smooth test function and set $\phi_{j}(t)=\phi\left(x_{j}, t\right)$. We multiply (2.1) by $\phi_{j} h$, sum, and integrate to obtain

$$
\left.\sum v_{j} \phi_{j} h\right|_{t_{1}} ^{t_{2}}-\int_{t_{1}}^{t_{2}} \sum v_{j} \dot{\phi}_{j} h d t+\int_{t_{1}}^{t_{2}} \sum u_{k} \delta \phi_{k} h d t=0 .
$$

We need to show that each term in (3.18) converges to the correspoding term in (1.14). Indeed, the equalities

$$
\begin{aligned}
\int_{t_{1}}^{t_{2}} \int_{0}^{\infty} u^{h} \phi_{x} d x d t & =\int_{t_{1}}^{t_{2}} \sum_{j} \int_{x_{j}}^{x_{j+1}} u^{h} \phi_{x} d x d t \\
& =\int_{t_{1}}^{t_{2}}\left(\sum\left(u_{k}+O\left(h^{1 / 2}\right)\right)\left(\delta \phi\left(x_{k}\right)+O(h)\right) h\right) d t \\
& =\int_{t_{1}}^{t_{2}} \sum u_{k} \delta \phi_{k} h d t+O\left(h^{1 / 2}\right)
\end{aligned}
$$

by (3.7), this proved for the last term in (3.18). The other terms in (3.18) can be treated in the same way. Take the test function $\varphi$ as in Sect. 1, and abbreviate the same with above, multiplying now $(2.2)$ by $\varphi_{k} h$, summing over $k$, and integrating with respect to $t$, arrive at:

$$
\begin{aligned}
& \left.\sum u_{k} \varphi_{k} h\right|_{t_{1}} ^{t_{2}}-\int_{t_{1}}^{t_{2}} \sum u_{k} \dot{\varphi}_{k} h d t-\int_{t_{1}}^{t_{2}} \sum p_{j} \delta \varphi_{j} h d t \\
& =-\int_{t_{1}}^{t_{2}} \sum \frac{\delta u_{j}}{v_{j}} \delta \varphi_{j} h d t+-\int_{t_{1}}^{t_{2}} \sum v_{j} \delta(\delta(\varphi))_{j} h d t .
\end{aligned}
$$

and give the argument only for the most difficult term, which is the first term on the right.

$$
\begin{aligned}
\int_{t_{1}}^{t_{2}} \int_{0}^{\infty} \frac{u_{x}^{h}}{v^{h}} \varphi_{x} d x d t & =\int_{t_{1}}^{t_{2}} \sum_{k} \int_{x_{k}}^{x_{k+1}} \frac{u_{x}^{h}}{v^{h}} \varphi_{x} d x d t \\
& =\int_{t_{1}}^{t_{2}}\left(\sum\left(\delta u_{j}+O\left(h^{1 / 2}\right)\right)\left(v_{j}^{-1}+O\left(h^{1 / 2}\right)\right)\left(\delta \varphi\left(x_{j}\right)+O(h)\right) h\right) d t \\
& =\int_{t_{1}}^{t_{2}} \sum \frac{\delta u_{j}}{v_{j}} \delta \varphi_{j} h d t+O\left(h^{1 / 2}\right)
\end{aligned}
$$

by (3.8)-(3.9), thus showing that the first term on the right in (3.19) approaches the corresponding term in (1.15) as $h \rightarrow 0$. And the other terms can be handled in a same way.

And only leave to prove the regularity conditions (1.10) and (1.11). First, $u \in C\left((0, \infty) ; H^{1}([0, \infty))\right), v \in C\left((0, \infty) ; H^{2}([0, \infty))\right)$ by $(3.7)-(3.10),(3.12)$ and (3.15)-(3.17). Then the argument based on the uniform estimate (1.19) shows that:

$$
v_{x}(\cdot, t) \rightarrow v_{o x} \quad \text { weakly in } L^{2} \text { as } t \rightarrow 0
$$


To prove strong convergence in $(3.20)$, let $\zeta \in C_{0}^{2}((0, \infty))$ and take $\zeta_{x}$ as the test function in (1.14), we get

$$
\begin{aligned}
& \left.\int_{0}^{\infty} v \zeta_{x} d x\right|_{t_{1}} ^{t_{2}}-\int_{t_{1}}^{t_{2}} \int_{0}^{\infty}\left(v \zeta_{x t}-u \zeta_{x x}\right) d x d t=0 \\
& \left.\int_{0}^{\infty} v_{x} \zeta d x\right|_{t_{1}} ^{t_{2}}+\int_{t_{1}}^{t_{2}} \int_{0}^{\infty} u_{x} \zeta_{x} d x d t=0
\end{aligned}
$$

set $t_{1}=0$,

$$
\int_{0}^{\infty} v_{x}\left(x, t_{2}\right) \zeta(x) d x=\int_{0}^{\infty} v_{x}(x, 0) \zeta(x) d x-\int_{0}^{t_{2}} \int_{0}^{\infty} u_{x} \zeta_{x} d x d t
$$

let $\zeta$ approach $v_{x}\left(x, t_{2}\right)$ to obtain

$$
\int_{0}^{\infty} v_{x}\left(x, t_{2}\right)^{2} d x=\int_{0}^{\infty} v_{0 x}(x) v_{x}\left(x, t_{2}\right) d x-\int_{0}^{t_{2}} \int_{0}^{\infty} u_{x} v_{x x}\left(x, t_{2}\right) d x d t
$$

last let $t_{2} \rightarrow 0$, we obtain

$$
v_{x}(\cdot, t) \rightarrow v_{o x} \quad \text { strongly in } L^{2} \text { as } t \rightarrow 0 .
$$

and then $v(\cdot, t) \in C\left([0, \infty) ; H^{1}([0, \infty))\right) \cap C\left((0, \infty) ; H^{2}([0, \infty))\right)$. A similar argument yields $u(\cdot, t) \in C\left([0, \infty) ; L^{1}([0, \infty))\right) \cap C\left((0, \infty) ; H^{1}([0, \infty))\right)$.

\section{References}

[1] Anderson, D.-M., McFadden, G.-B., Wheller, A.-A.: Diffuse-interface methods in fluid mech. Ann. Rev. Fluid Mech. 30, C139-C165 (1998)

[2] Bresch, D., Desjardins, B., Lin, C.-K.: On some compressible fluid models: Korteweg, lubrication, and shallow water systems. Commun. Partial. Differ. Equ. 28(3-4), 843-868 (2003)

[3] Bona, J.L., Sachs, R.L.: Global existence of smooth solutions and stability of solitary waves for a generalized Boussinesq equation. Commun. Math. Phys. 118(1), C15-C29 (1988)

[4] Haspot, B.: Existence of global weak solution for compressible fluid models of Korteweg type. J. Math. Fluid Mech. (2009). doi:10.1007/s00021-009-0013-2

[5] Cahn, J.-W., Hilliard, J.-E.: Free energy of a nonuniform system, I. Interfacial free energy. J. Chem. Phys. 28, C258-C267 (1998)

[6] Danchin, R., Desjardins, B.: Existence of solutions for compressible fluid models of Korteweg type. Annales de l'IHP, Analyse Non linéaire 18, C97-C133 (2001)

[7] Dunn, J.-E., Serrin, J.: On the thermomechanics of interstitial working. Arch. Ration. Mech. Anal. 88(2), C95-C133 (1985)

[8] Gurtin, M.-E., Poligone, D., Vinals, J.: Two-phases binary fluids and immiscible fluids described by an order parameter. Math. Models Methods Appl. Sci. 6(6), C815-C831 (1996) 
[9] Hoff, D.: Construction of solutions for compressible, isentropic Navier-Stokes equations in one space dimension with non-smooth initial data. Proc. R. Soc. Edinburgh, Sect. A 103, 301-315 (1986)

[10] Hoff, D.: Global existence for 1D, compressible, isentropic Navier-Stokes equations with large initial data. Trans. Am. Math. Soc. 303(1), 169-181 (1987)

[11] Jamet, D., Lebaigue, O., Coutris, N., Delhaye, J.-M.: The second gradient method for the direct numerical simulation of liquid-vapor flows with phase change. J. Comput. Phys. 169(2), C624-C651 (2001)

[12] Kato, T.: Quasi-linear equations of evolution, with applications to partial differential equations. In Spectral theory and differential equations. In: Proc. Sympos., Dundee, 1974; dedicated to Konrad Jorgens, pp. C25-C70. Lecture Notes in Math., vol. 448. Springer, Berlin (1975)

[13] Korteweg, D.-J.: Sur la forme que prennent les équations du mouvement des fluides si l'on tient compte des forces capillaires par des variations de densité. Arch. Néer. Sci. Exactes Sér. II 6, C1-C24 (1901)

[14] Rowlinson, J.-S.: Translation of J.D van der Waals The thermodynamic theory of capillarity under the hypothesis of a continuous variation of density. J. Stat. Phys. 20(2), C197-C244 (1979)

[15] Truedelland, C., Noll, W.: The nonlinear field theories of mechanics, 2nd edn. Springer, Berlin (1992)

[16] Tsyganov, E.: Global existence and asymptotic convergence of weak solutions for the one-dimensional Navier-Stokes equations with capillarity and nonmonotonic pressure. J. Differ. Equ. 245, 3936-3955 (2008)

Zhong Tan and Rongcong Guo

School of Mathematical Sciences, Xiamen University,

Xiamen 361005, Fujian, China

e-mail: g.rongcong@foxmail.com

Zhong Tan

e-mail: ztan85@163.com

Received: 9 June 2010.

Accepted: 1 February 2011. 